\title{
Effect of Ocean Spray on Vertical Momentum Transport Under High-Wind Conditions
}

\author{
Yevgenii Rastigejev • Sergey A. Suslov • Yuh-Lang Lin
}

Received: 4 October 2010 / Accepted: 17 May 2011 / Published online: 17 June 2011

(C) The Author(s) 2011. This article is published with open access at Springerlink.com

\begin{abstract}
Two mathematical models are proposed detailing the influence of ocean spray on vertical momentum transport under high-wind conditions associated with a hurricane or severe storm. The first model is based on a turbulent kinetic energy (TKE) equation and accounts for the so-called lubrication effect due to the reduction of turbulence intensity. The second model is based on Monin-Obukhov similarity (MOS) and uses available experimental data. It is demonstrated that the flow acceleration is negligible for wind speeds below a certain critical value due to the fact that the spray volume concentration is low for such speeds. For wind speeds higher than the critical value, the spray concentration rapidly increases, which results in significant flow acceleration. Both models produce qualitatively similar results for all turbulent flow parameters considered. It was found that the MOS-based model tends to predict a noticeably stronger lubrication effect than the TKE-based model, especially for lower wind speeds. The results of model calculations are in very good agreement with available experimental data for the spray production values near the upper bound. It is also shown that neither the value of the turbulent Schmidt number in the TKE-based model nor the choice of a stability profile function affects the spray-laden flow dynamics significantly.
\end{abstract}

Keywords Air-ocean drag $\cdot$ Hurricanes $\cdot$ Ocean spray $\cdot$ Stratified turbulent flows

\footnotetext{
Y. Rastigejev

Department of Mathematics, North Carolina A\&T State University, Greensboro, NC, USA

Y. Rastigejev $(\varangle) \cdot$ Y.-L. Lin

Department of Energy and Environmental Systems, North Carolina A\&T State University, 302 Gibbs Hall, 1601 E. Market Street, Greensboro, NC 27411, USA

e-mail: yarastig@ncat.edu
}

\section{S. A. Suslov}

Mathematics H38, FEIS, Swinburne University of Technology, Hawthorn,

VIC 3156, Australia

Y.-L. Lin

Department of Physics, North Carolina A\&T State University, Greensboro, NC, USA 


\section{Introduction}

Accurate modelling of ocean spray is important for understanding strong storm and tropical cyclone dynamics. It has been pointed out (Bortkovskii 1973; Borisenkov 1974; Ling and Kao 1976; Lighthill 1999) that ocean spray produced via different physical mechanisms (Andreas et al. 1995; Andreas and DeCosmo 1999; Kudryavtsev 2006) significantly affects these dynamics. The spray influences the intensity and structure of a hurricane both mechanically and thermodynamically. Mechanical mixing of spray with the near-surface airflow has a strong effect on the momentum exchange between the atmosphere and the upper sea layer. At the same time, as discussed, for example, by Kepert et al. (1999), Andreas and Emanuel (2001), Gall et al. (2008), the ocean spray injected into the air leads to significant variations of sensible and latent heat fluxes in the near-surface layer. This enhanced heat exchange is responsible for the creation of stable or unstable vertical thermal stratification, which in turn affects mechanical flow characteristics and the turbulence level within the layer. Such a twoway coupling between mechanical and thermodynamic influences of the ocean spray is an inherent feature of air-sea exchange (Fairall et al. 1994), yet the ultimate influence of spray evaporation on hurricane dynamics remains the subject of heated debate. A good review of the state of affairs in this area is given by Wang et al. (2001) who concluded that 'further research is needed to determine which (model) most reliably represents reality, as to whether spray would cool or warm the surface layer', and thus would lead to either suppression or intensification of a hurricane.

In the present study, we choose to take a different line of study. We aim to investigate the pure mechanical influence of sea spray on the level of turbulence and wind speed in the near-surface atmospheric layer, assuming that a mechanical (mixing) effect of the spray on the averaged characteristics of a near-sea atmospheric layer is much stronger than the thermodynamic influence of the spray on the airflow. Using such an assumption, we will decouple the problem and consider the mechanical characteristics of the airflow independently of its thermodynamic properties. Indirectly, such an approach is warranted by studies of, for example, Fairall et al. (1994), Kepert et al. (1999) and Wang et al. (2001), which indicate that the evaporation of sea spray largely redistributes sensible and latent heat fluxes in the marine boundary layer rather than alters the net enthalpy flux that affects the hurricane intensity. Therefore, at the coarsest mechanical model level, the details of thermodynamic processes can be neglected.

Various mechanical factors that contribute to the air-sea momentum exchange have been investigated in a number of recent studies: Donelan et al. (2004), Makin (2004), Barenblatt et al. (2005), Kudryavtsev (2006), Vakhguelt (2007), Babanin and Makin (2008) to name a few. Several approaches have been proposed to estimate the effect of sea spray on the wind stress (Makin 2004; Barenblatt et al. 2005; Kudryavtsev 2006). Makin (2004) assumed a limiting saturation in a thin region adjacent to the sea surface as described previously by Barenblatt and Golitsyn (1974) for high flow speeds: the air in this layer absorbs the maximum possible amount of droplets given the unrestricted production of ocean spray. Makin (2004) reported the possibility of significant acceleration of airflow in this regime. Although the author presented an interesting theoretical paradigm, the spray production is primarily defined by the wind speed (Andreas 1998), and further study is required to establish whether the air motion can be assumed sufficiently fast for the regime of limiting saturation to be observed in reality. Moreover, the regime of limiting saturation may occur only for spray droplets characterized by a relatively small terminal fall velocity (Barenblatt and Golitsyn 1974). It is unclear though whether a significant number of such droplets are present in ocean spray. 
Kudryavtsev (2006) presented an alternative model of the spray-laden atmospheric boundary layer that accurately predicts a decrease in the 10-m drag coefficient $C_{\mathrm{d}}$ for wind speeds in the range $30-45 \mathrm{~m} \mathrm{~s}^{-1}$. However, comparison with the experimental data of Powell et al. (2003) indicates that the model might overestimate the degree of reduction in the drag coefficient for flow speeds above $45 \mathrm{~m} \mathrm{~s}^{-1}$ and the value of friction velocity at smaller wind speeds.

Barenblatt et al. (2005, BCP hereafter) recently reported that ocean spray leads to a significant decrease in the turbulence intensity of atmospheric flow, which in turn reduces the air-sea drag and results in near-surface flow acceleration. The model presented in BCP is based on a sound theory of sediment-laden flows developed earlier (Barenblatt 1953; Kolmogorov 1954). The numerical calculations reported in BCP suggest almost an order of magnitude increase in wind speed when very large droplets are present in the flow. In an attempt to confirm these results, we implemented BCP's model independently, but were not able to reproduce them in our calculations; instead, our numerical results predict a much smaller increase in wind speed indicating a likely numerical error in BCP.

A large number of various hurricane and air-sea interaction models are available in the literature, some including a very detailed and specific description of the influencing factors and realistic regional geographic features, e.g., a large hybrid model of hurricane Opal in the Gulf of Mexico suggested by Bao et al. (2000). Yet, the conclusion made by the authors there was that a realistic hurricane evolution could be simulated without a "physically faithful description of fluxes at the air-sea interface, so long as the amount of momentum and enthalpy fluxes from the model's lower boundary ... is sufficient to sustain hurricane evolution'. Therefore, the aim of the present study is to suggest a physically meaningful, yet easy-to-use model, which would be able to provide a good approximation for such fluxes.

We explore two approaches describing spray-laden turbulent boundary-layer dynamics. The first model considers the coupled vertical momentum, ocean spray and turbulent kinetic energy transports (TKE model). It is impossible to account accurately for the contraction of turbulent eddies due to the spray stratification within the framework of the TKE model. Therefore, based on Soulsby and Wainwright (1987), we apply Monin-Obukhov similarity (MOS) theory for a sediment-stratified boundary layer and use the analogy between thermally stratified and sediment-stratified boundary layers when developing our model. We consider two stability functions for momentum profile measurements in the thermally stratified stable atmospheric layer: the 'classical' log-linear function (Dyer 1974) and the function recently obtained by Grachev et al. (2007). Both approaches have their advantages and disadvantages. The TKE-based model is derived from the fundamental principles of turbulent flow theory, but it cannot consistently account for such effects as the shrinking of turbulent scales in a thermally or sediment-stratified boundary layers. The MOS model is based on a semi-empirical approach that uses experimentally obtained stability functions for the mean flow velocity and temperature (thermally-stratified boundary layer) or sediment (sediment-stratified boundary layer) profiles.

We show that at high wind speeds the spume spray, which is produced by tearing the droplets directly from the wave crests, primarily affects the turbulent momentum transport. The spray concentration is determined by the generation rate that is a function of the wind speed: the available experimental data indicate that the spume-spray generation rate increases exponentially with the flow speed (Monahan 1986; Wu 1993; Andreas 1998). Unfortunately, there exists a large degree of uncertainty regarding the actual rate values, especially for high winds. Andreas (1998) reported the difference in the spray production rates suggested by different authors of up to six orders of magnitude. For example, the spray production rate reported by Andreas (1998) for an airflow speed of $35 \mathrm{~m} \mathrm{~s}^{-1}$ differs from that of Wu (1993) 
by four orders of magnitude. For the purpose of our investigation, we estimated the upper and lower bounds for the exponential growth rate for the spume-spray generation (the weakest and the strongest spray impact regimes, respectively) based on the available experimental data (Lai and Shemdin 1974; Monahan 1986; De Leeuw 1993; Smith et al. 1993; Wu 1993; Anguelova et al. 1999). We found that spume generated at the highest detected growth rate induces a lubrication effect at a noticeably lower flow speed (of about $30 \mathrm{~m} \mathrm{~s}^{-1}$ ) than that at the lowest growth rate (of about $50 \mathrm{~m} \mathrm{~s}^{-1}$ ).

We found that both TKE and MOS-based models produce qualitatively similar results, although the MOS-based model tends to predict a noticeably stronger lubrication, especially for lower flow-speed values. On the other hand, the TKE model predicts a somewhat stronger lubrication for smaller turbulent Schmidt numbers. The choice of stability functions does not significantly affect the strength of the lubrication effect in MOS-based models. This article is organized as follows: Sect. 2 presents the derivation of a model and discusses its parametric validity range. Numerical and analytical results are discussed in Sect. 3. Conclusions are presented in Sect. 4.

\section{Mathematical Model of Ocean Spray}

\subsection{Governing Equations}

The continuity equation for the distribution of droplets suspended in a horizontally homogeneous layer of air can be written as (Monin and Yaglom 2007)

$$
-\frac{\mathrm{d}}{\mathrm{d} z}\left[\alpha_{0 \mathrm{~s}} k \frac{\mathrm{d} n}{\mathrm{~d} z}+a_{0} n\right]=q_{0},
$$

where $n(z ; r), q_{0}(z ; r)$ and $a_{0}(r)$ are the droplet volume mixing ratio, the source function and the terminal velocity of the droplet, respectively (all depending on the height $z$ above the averaged water surface level and parametrized by the droplet radius $r$ ), $k$ is the turbulent eddy diffusivity and $\alpha_{0 \text { s }}$ is the reciprocal of the turbulent Schmidt number (Monin and Yaglom 2007), which is the ratio of the eddy viscosity and the eddy mass diffusivity. After integrating (1) between an arbitrary value of $z$ and infinity, and assuming that the spray concentration is negligible at infinity (which implies that $\left.n\right|_{z \rightarrow \infty}=0$ and $\left.\frac{\mathrm{d} n}{\mathrm{~d} z}\right|_{z \rightarrow \infty}=0$ ) we obtain

$$
\alpha_{0 \mathrm{~s}} k \frac{\mathrm{d} n}{\mathrm{~d} z}+a_{0} n=q
$$

where

$$
q(r ; z)=\int_{z}^{\infty} q_{0}\left(z^{\prime} ; r\right) \mathrm{d} z^{\prime} .
$$

Experimental data on ocean spray production and the theoretical description of relevant physical mechanisms were previously presented by a number of authors, e.g. Wu (1979), Andreas et al. (1995). We briefly summarize them below.

It is known that ocean spray is generated from three sources: jet droplets, film droplets and spume droplets (Andreas and DeCosmo 1999). The breaking of ocean waves causes an entrainment of air, which results in whitecap formation. Film droplets occur as a result of bursting whitecap bubbles, while jet droplets are ejected from the centres of collapsing 
bubble cavities. Jet droplets and film droplets (whose radii range from 0.5 to $50 \mu \mathrm{m}$ ) form bubble-generated spray, whose rate of production is determined by the rate at which air is entrained into the ocean surface layer. It is proportional to the rate of whitecap formation. Observations indicate that bubble-generated droplets tend to be injected upwards. The height of injection is under $0.2 \mathrm{~m}$ (Wu 1979). Therefore, the bubble-generated droplets are confined to a thin layer near the surface. A spume spray is generated via the tearing of water droplets off the wave crests. Spume droplets typically have radii larger than $20 \mu \mathrm{m}$ and are ejected horizontally. Their source is confined to a thin layer above the major wave crests.

It is intuitively clear that the volume fraction of a spume spray should increase with the wind speed. Experimental results indicate that the mass of a spume spray is indeed larger than that of jet and film spray for $10-\mathrm{m}$ wind speeds $u_{10}>12-13 \mathrm{~m} \mathrm{~s}^{-1}$ (De Leeuw 1986). As noted by Andreas (1998), the compilation of spray-generation functions (SGFs) from previous studies (Blanchard 1963; Ling et al. 1980; Gathman 1982; Monahan 1986; Bortkovskii 1987; Woolf et al. 1987; Iida et al. 1992; Wu 1992, 1993; Smith et al. 1993) supports this conclusion. It appears that the spume-spray production rate is at least an order (and possibly two to three orders) of magnitude larger than the bubble-generated spray production for $u_{10}>20$ $\mathrm{m} \mathrm{s}^{-1}$. Therefore, spume spray, rather than bubble-generated spray, dominates the vertical momentum transport in the flow at high wind speeds. Since spume spray is produced in a relatively thin layer just above the wave crests we represent the source for the spume-spray generation by the Dirac $\delta$-function (Fairall et al. 2009):

$$
q_{0}(z ; r)=Q_{0}(r) \delta\left(z-z_{\mathrm{W}}\right),
$$

where $Q_{0}(r)$ is the strength of the spray-production source also known as the spray-generation function and $z \mathrm{~W}$ is the average height of the wave crests. From (3) and (4), we obtain the following expression for the source term $q(z ; r)$ :

$$
q(z ; r)=Q_{0}(r) H(z \mathrm{~W}-z),
$$

where $H(z)$ is the Heaviside step function, which implies that the source term $q(z ; r)$ is equal to the value of $Q_{0}(r)$ below $z \mathrm{~W}$ and zero above $z \mathrm{~W}$. Therefore, Eq. (2) can be rewritten as

$$
\begin{aligned}
& \alpha_{0 \mathrm{~s}} k \frac{\mathrm{d} n}{\mathrm{~d} z}+a_{0} n=Q_{0}, \quad z<z_{\mathrm{W}}, \\
& \alpha_{0 \mathrm{~s}} k \frac{\mathrm{d} n}{\mathrm{~d} z}+a_{0} n=0, \quad z>z_{\mathrm{W}} .
\end{aligned}
$$

It is noted that the coefficient of turbulent diffusion $k$, which depends on the vertical coordinate $z$, becomes zero in the thin layer near the sea surface at $z=0$ where turbulence is suppressed by viscosity. After substituting $k=0$ into Eq. (6), we obtain the following boundary condition for this equation at $z=0$ :

$$
n(r)=\frac{Q_{0}(r)}{a_{0}(r)} .
$$

The general solution of (6) can be written in the form:

$$
n(z)=\frac{Q_{0}}{a_{0}}+B \exp \left(-\frac{a_{0}}{\alpha_{0 \mathrm{~s}}} \int^{z} \frac{\mathrm{d} \xi}{k(\xi)}\right),
$$

where $B$ is some constant. After applying the boundary condition (8)-(9), we obtain that $B=0$, i.e., the spray concentration does not depend on the vertical coordinate for $z<z \mathrm{~W}$ and is equal to (8). In order to solve Eq. (7), we need to know the dependence of $Q_{0}$ and 
$\alpha_{0 \mathrm{~s}}$ on the droplet radius $r$. Unfortunately, there are no reliable experimental data for $\alpha_{0 \mathrm{~s}}(r)$ and $Q_{0}(r)$ available for high wind speeds $u_{10}>30 \mathrm{~m} \mathrm{~s}^{-1}$. Therefore, we consider a simpler model that deals with the total volume mixing ratio $s(z)$ rather than with the size-dependent distribution $n(z ; r)$. The total volume mixing ratio $s(z)$ can be expressed in terms of the size-dependent volume mixing ratio distribution $n(z ; r)$ as

$$
s(z)=\int_{0}^{\infty} n(z ; r) \mathrm{d} r .
$$

From (8) and (10), we conclude that the total spray volume mixing ratio $s(z)$ below $z \mathrm{~W}$ takes a constant value

$$
s_{0}=\int_{0}^{\infty} \frac{Q_{0}(r)}{a_{0}(r)} \mathrm{d} r, \quad z<z \mathrm{~W} .
$$

Next, by integrating Eq. (7) with respect to the droplet radius $r$, we obtain

$$
k \frac{\mathrm{d}\left(\alpha_{\mathrm{S}} s\right)}{\mathrm{d} z}+a s=0, \quad z>z_{\mathrm{W}},
$$

where $a$ and $\alpha_{\mathrm{S}}$ are, respectively, the mean terminal velocity and reciprocal Schmidt number averaged over the complete spectrum of droplet sizes:

$$
\begin{aligned}
a(z) & =\frac{1}{s(z)} \int_{0}^{\infty} a_{0}(r) n(z ; r) \mathrm{d} r, \\
\alpha_{\mathrm{s}}(z) & =\frac{1}{s(z)} \int_{0}^{\infty} \alpha_{0 \mathrm{~s}}(r) n(z ; r) \mathrm{d} r .
\end{aligned}
$$

The value of the mean terminal velocities $a_{\mathrm{b}}$ and $\alpha_{\mathrm{bs}}$ below the crest level at $z<z_{\mathrm{W}}$ is found by substituting (8) into (13) and (14)

$$
\begin{aligned}
a_{\mathrm{b}} & =\frac{1}{s_{0}} \int_{0}^{\infty} Q_{0}(r) \mathrm{d} r, \\
\alpha_{\mathrm{bs}} & =\frac{1}{s_{0}} \int_{0}^{\infty} \frac{Q_{0}(r) \alpha_{0 \mathrm{~s}}(r)}{a_{0}(r)} \mathrm{d} r,
\end{aligned}
$$

where $s_{0}$ is given by (11). The values of the average terminal velocity $a(z)$ and the average reciprocal Schmidt number $\alpha_{\mathrm{S}}(z)$ defined by (13) and (14), respectively, depend on the spray distribution function $n(z ; r)$, which is not known. Therefore, in order to solve (12), we need to make reasonable assumptions about the distributions of $a(z)$ and $\alpha_{\mathrm{s}}(z)$. Specifically, we assume that the average terminal velocity $a(z)$ above the wave crests is equal to the average terminal velocity $a_{\mathrm{b}}$ below the wave crests. This assumption is justified by the following arguments. The average terminal velocity is determined largely by droplets with sizes in a relatively narrow range of $r=100-200 \mu \mathrm{m}$ (Andreas 1998; Wu 1993). This is so because the volume spray production rate is only large for such droplets. The droplet terminal velocity, however, does not vary significantly over the considered range of sizes and remains of the same order of magnitude as the mean terminal velocity, $\approx 1 \mathrm{~m} \mathrm{~s}^{-1}$ (more specifically, 
$\left.a(r)=0.7-1.5 \mathrm{~m} \mathrm{~s}^{-1}\right)$. Therefore, the difference between the spray distributions above and below wave crests should not cause a significant variation in the average droplet terminal velocity $a(z)$.

\subsection{Application of the TKE Theory to the Spray-Laden Boundary Layer}

The remaining equations for the momentum exchange and turbulent kinetic energy (TKE) balance can be written in the following form (Barenblatt 1996; Monin and Yaglom 2007):

$$
\begin{array}{r}
k \frac{\mathrm{d} u}{\mathrm{~d} z}=u_{*}^{2}, \\
k\left(\frac{\mathrm{d} u}{\mathrm{~d} z}\right)^{2}(1-K o)-\varepsilon=0,
\end{array}
$$

where $u$ is the wind speed (dependent variable) and $u_{*}$ is the friction velocity (parameter). The eddy viscosity $k$, Kolmogorov number $K o$ and the TKE dissipation rate $\varepsilon$ are expressed in terms of $u, s$ (the third dependent variable), turbulence kinetic energy $e$, and turbulence mixing length $l$ as

$$
\begin{aligned}
k & =l \sqrt{e}, \\
K o & =-\frac{\alpha_{\mathrm{s}} \sigma g}{u_{z}^{2}} \frac{\mathrm{d} s}{\mathrm{~d} z}, \\
\varepsilon & =\frac{e^{3 / 2}}{l},
\end{aligned}
$$

where $\sigma=\left(\rho_{\mathrm{w}}-\rho_{\mathrm{a}}\right) / \rho_{\mathrm{a}} \approx 10^{3}, \rho_{\mathrm{a}}$ and $\rho_{\mathrm{w}}$ are the densities of air and seawater, respectively, and $g$ is the gravitational acceleration. Note that the Kolmogorov number $K o$ for a spray-laden atmospheric boundary layer is analogous to the flux Richardson number $R f$ for a thermally stratified boundary layer (Monin and Yaglom 2007). We adopt the following expression for the mixing length (Barenblatt 1996):

$$
l=k_{\infty} z \Phi(K o)
$$

where $k_{\infty}=0.4$ (Tennekes 1973) is a non-dimensional parameter that depends on Reynolds number $R e$ (Barenblatt 1996) and is taken in the limit of $R e \rightarrow \infty$, and $\Phi(K o)$ is a function of the Kolmogorov number that accounts for the possible contraction of the turbulence mixing length due to the atmospheric stratification. Based on BCP, we assume $\Phi(K o)=\Phi(0)=1$. It is noted that this model is valid for small values of $s \sigma$, i.e. for $s<10^{-4}$. For larger values of this product, the spray inertia might become important. To keep the current analysis reasonably short, we omit reporting the analysis of an extended model that accounts for inertia to a separate publication.

\subsection{Application of Monin-Obukhov Similarity Theory to the Spray-Laden Boundary Layer}

We follow the Soulsby and Wainwright (1987) approach that assumes that MOS can be used for the sediment-laden turbulent boundary layer. The Obukhov length $L_{\mathrm{S}}$ (Businger and Yaglom 1971), also referred to as the Monin-Obukhov length, for the layer stratified by the ocean spray is defined as

$$
L_{\mathrm{s}}=\frac{u_{*}^{3}}{k_{\infty} a s \sigma g} .
$$


According to the MOS theory the following universal similarity laws should hold

$$
\begin{aligned}
\frac{\mathrm{d} u}{\mathrm{~d} z} & =\frac{u_{*}}{k_{\infty} z} \phi_{\mathrm{m}}(\xi), \\
\frac{\mathrm{d} s}{\mathrm{~d} z} & =\frac{a s}{k_{\infty} u_{*} z} \phi_{\mathrm{h}}(\xi),
\end{aligned}
$$

where

$$
\xi(z) \equiv \frac{z}{L_{\mathrm{S}}}
$$

where $\phi_{\mathrm{m}}$ and $\phi_{\mathrm{h}}$ are non-dimensional vertical gradients (also referred to as stability profile functions) of the mean wind speed $u$ and the mean value of an active scalar, which is the temperature distribution for a thermally stratified boundary layer or the spray concentration profile $s$ in a spray-laden boundary layer. Even though the gradient Richardson number

$$
R i=\frac{\xi \phi_{\mathrm{h}}(\xi)}{\phi_{\mathrm{m}}^{2}(\xi)}
$$

is a commonly used indicator of boundary-layer stability, following Grachev et al. (2005), we use the value of the parameter $\xi$ for this purpose. Traditionally, three distinct regimes are distinguished for the stable boundary layer (Mahrt 1998): weakly stable $(0<\xi \lesssim O(0.1))$, transitional $(O(0.1) \lesssim \xi \lesssim O(1))$ and very stable $(\xi \gtrsim O(1))$. The weakly stable boundary layer is described by the classical MOS theory. Shortly, we demonstrate that the transitional and very stable layer (at least up to some value of $R i$ ) can also be described by the generalized MOS theory that uses scaling variables redefined in terms of the $z$-dependent local fluxes and generalized expressions for the stability functions $\phi_{\mathrm{m}}(\xi)$ and $\phi_{\mathrm{h}}(\xi)$. It is noted that the critical Richardson number $R i_{\mathrm{c}} \approx 0.2$ corresponds to the lower part of the very stable boundary-layer parametric range $(O(1) \lesssim \xi \lesssim O(10))$.

The classical expressions for the stability functions,

$$
\phi_{\mathrm{m}}(\xi)=\phi_{\mathrm{h}}(\xi)=1+\beta \xi,
$$

with $\beta \approx 5$, originally suggested by Businger et al. (1971), were based on the analysis of measurements in a thermally stratified atmospheric boundary layer. These expressions are referred to as the log-linear distributions, and were adapted for sediment-laden flows by e.g. Soulsby and Wainwright (1987), Wamser and Lykossov (1995), and Kudryavtsev (2006). However, the experimental data (Businger et al. 1971; Dyer 1974; Yaglom 1977; King 1990; Beljaars and Holtslag 1991; Howell and Sun 1999; Grachev et al. 2008) fit the log-linear expressions well only for small values of $\xi<0.1$ (weakly stable boundary layer). Nieuwstadt (1984) extended the classical MOS theory by considering scales that depend on the vertical coordinate $z$ (local scaling) and successfully applied it to a stable nocturnal thermally-stratified atmospheric boundary layer. It was shown that the log-linear distribution works well if local scaling is used for both the weakly stable and transitional regimes $(\xi \lesssim O(1))$ i.e. if the ocean-spray volume fraction is relatively small, $s<s_{\text {lim }}$. In order to estimate $s_{\text {lim }}$, then we use definitions (21) and (24) of $L_{\mathrm{S}}$ and $\xi$ to obtain

$$
s_{\lim }=\frac{u_{*}^{3}}{k_{\infty} a \sigma g z} .
$$

For typical values of parameters $z \approx 10 \mathrm{~m}, a \approx 1 \mathrm{~ms}^{-1}, \sigma \approx 10^{3}, g \approx 10 \mathrm{~m} \mathrm{~s}^{-1}$, $u_{*} \approx 1 \mathrm{~m} \mathrm{~s}^{-1}$ we obtain $s_{\text {lim }} \sim 10^{-5}$. 
Table 1 The values of $\xi$ and $R i$ at wave-crest level for $u_{10}=50 \mathrm{~m} \mathrm{~s}^{-1}$ for different values of $\delta$

\begin{tabular}{lll}
\hline$\delta$ & $\xi$ & $R i$ \\
\hline 0.2 & $3.5 \times 10^{-2}$ & $3 \times 10^{-2}$ \\
0.4 & 0.8 & 0.14 \\
0.6 & 3.3 & 0.19 \\
0.8 & 8.5 & 0.26 \\
\hline
\end{tabular}

Recent experiments (Grachev et al. 2007) indicate that the stability function $\phi_{\mathrm{m}}(\xi)$ increases with $\xi$ significantly slower than that predicted by the log-linear distribution (26) for $\xi>1$ (very stable boundary layer). Therefore, the log-linear distribution is not applicable for concentrations of the ocean spray higher than $s \sim 10^{-5}$. Various stability profile functions have been suggested in the literature in an attempt to extend the MOS applicability to larger Ri numbers (Lettau 1957; Businger et al. 1971; Dyer 1974; Holtslag and de Bruin 1988). Recently, Grachev et al. (2007) proposed a different analytical form for the stability functions that are based on the analysis of comprehensive flux-profile data from the Surface Heat Budget of the Arctic Ocean experiment (SHEBA)

$$
\begin{aligned}
\phi_{\mathrm{m}}(\xi) & =1+\frac{6.5 \xi(1+\xi)^{1 / 3}}{1.3+\xi}, \\
\phi_{\mathrm{h}}(\xi) & =1+\frac{5 \xi+5 \xi^{2}}{1+3 \xi+\xi^{2}} .
\end{aligned}
$$

These expressions are based on comprehensive long-term multilevel measurements and appear to be the most reliable among all the stability functions reported in the literature to date. Grachev et al. (2007) showed experimentally that they are valid for values of $\xi$ up to 100 and $R i$ up to 0.62 , which corresponds to $s \approx 10^{-3}$ for the spray-stratified flow. Therefore, the generalized MOS theory (with modified expressions for the stability functions (28) and (29) and the $z$-dependent local scaling) remains applicable even for the very stable regime. Numerical calculations that we report in the article cover the range of experimental data available for wind speeds $u_{10}<50 \mathrm{~m} \mathrm{~s}^{-1}$. We found that the values of $\xi\left(z_{\mathrm{W}}\right)$ and $R i\left(z_{\mathrm{W}}\right)$ at the wave-crest level, $z=z_{\mathrm{W}}$, do not exceed the respective maxima of 8.5 and 0.26 , (see Table 1) achieved for the upper-bound spray-production function $(\delta=0.8$ ) and the flow speed of $50 \mathrm{~m} \mathrm{~s}^{-1}$ (details are given in Sect. 3.1). Therefore, we remain safely in the validity range of the generalized MOS theory and adopt relations (28) and (29) for our present calculations and compare the obtained numerical results with the ones for log-linear stability functions previously used for sediment-laden flow modelling.

\subsection{Boundary Conditions}

It was shown experimentally (Wu 1980) that the surface roughness length $z_{0}$ is determined by (Charnock 1955)

$$
z_{0}=\frac{c_{0} u_{*}^{2}}{g},
$$

where $c_{0}=0.018$. For a typical value of $u_{*}=1 \mathrm{~m} \mathrm{~s}^{-1}$, we obtain $z_{0} \approx 1.8 \times 10^{-3} \mathrm{~m}$. Since the total spray-volume fraction $s=s_{0}$ is constant for $z<z_{\mathrm{W}}$, from (19b), we obtain that $K o=0$ and the TKE equation (18) takes a simple form $\varepsilon=k(\mathrm{~d} u / \mathrm{d} z)^{2}$ in this region. 
Therefore, we obtain the following expression for the TKE from the momentum exchange Eq. (17) and expressions (19a) and (19c) for $k$ and $\varepsilon$ :

$$
e=u_{*}^{2}, \quad z<z \mathrm{~W} .
$$

Clearly, spray distributed uniformly in the vertical direction does not affect the turbulence intensity. Subsequently, from (17), (19a), (19b), (20) and (31), we obtain the logarithmic profile for the flow-speed distribution

$$
u=\frac{u_{*}}{k_{\infty}} \ln \left(\frac{z}{z_{0}}\right), \quad z<z \mathrm{~W} .
$$

Then the flow speed at the wave crest level $z=z_{\mathrm{W}}$ is

$$
u_{\mathrm{W}}=\frac{u_{*}}{k_{\infty}} \ln \left(\frac{z}{z_{0}}\right) .
$$

The ocean-spray generation rate $Q_{0}(r)$ can be expressed as a function of the reference wind speed $u_{10}$ (Wu 1993; Andreas 1998), and so the total spray volume fraction $s_{0}$ is also a function of $u_{10}$ on account of (11):

$$
s_{0}=f\left(u_{10}\right)
$$

Detailed information regarding the function $f\left(u_{10}\right)$ is provided in Sect. 2.6. Relations (33) and (34) serve as the boundary conditions for the momentum and ocean-spray mass exchange equations.

\subsection{Non-Dimensional Form of the Governing Equations}

Based on BCP, we introduce the non-dimensional variables and parameters $Z=z / L_{\mathrm{r}}$, $Z_{\mathrm{w}}=z_{\mathrm{w}} / L_{\mathrm{r}}, Z_{0}=z_{0} / L_{\mathrm{r}}, L=l / L_{\mathrm{r}}, U=u / u_{*}, E=e / u_{*}^{2}, \omega=a /\left(\alpha_{\mathrm{s}} k_{\infty} u_{*}\right)$, where the reference length scale $L_{\mathrm{r}}=u_{*}^{2} /\left(\alpha_{\mathrm{S}} \sigma g\right)=u_{*}^{3} k_{\infty} \omega /(a \sigma g)$. The non-dimensional form of the governing equations (12), (17) and (18) then becomes

$$
\begin{aligned}
K \frac{\mathrm{d} U}{\mathrm{~d} Z} & =1, \\
K \frac{\mathrm{d} s}{\mathrm{~d} Z}+k_{\infty} \omega s & =0, \\
E^{2}+K o-1 & =0,
\end{aligned}
$$

where

$$
\begin{aligned}
K & =L \sqrt{E}, \\
L & =k_{\infty} Z \Phi(K o), \\
K o & =k_{\infty} \omega L \sqrt{E} s .
\end{aligned}
$$

Boundary conditions (33) and (34) take the following non-dimensional forms:

$$
\begin{aligned}
& U\left(Z_{\mathrm{W}}\right)=U_{\mathrm{W}} \equiv \frac{1}{k_{\infty}} \ln \left(\frac{Z_{\mathrm{W}}}{Z_{0}}\right), \\
& s\left(Z_{\mathrm{W}}\right)=s_{0}=f\left(u_{*} U_{10}\right),
\end{aligned}
$$


Fig. 1 A conceptual diagram of spume generated by the wind tearing off the wave crests: $z_{0}$ and $z_{\mathrm{W}}$ are the roughness length and the heights of wave crests, respectively. Ocean spray is distributed uniformly below the wave crests

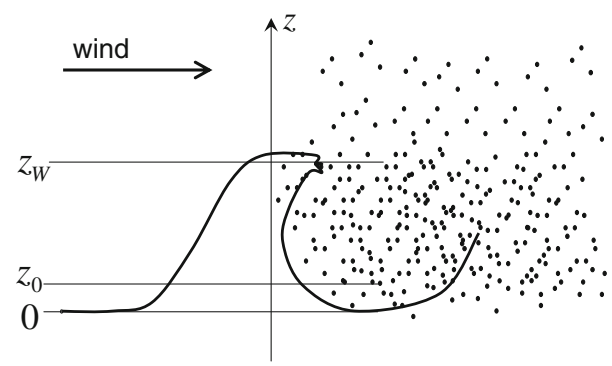

where $U_{10}$ is the $10-\mathrm{m}$ non-dimensional flow speed. We use the standard definition of the 10 -m drag coefficient,

$$
C_{\mathrm{d}}=\left(\frac{u_{*}}{u_{10}}\right)^{2}=\frac{1}{U_{10}^{2}} .
$$

The effective turbulent roughness scale $z_{0, \mathrm{e}}$ is defined assuming the logarithmic flow-speed profile:

$$
U=\frac{1}{k_{\infty}} \ln \left(\frac{z}{z_{0, \mathrm{e}}}\right)
$$

so that

$$
z_{0, \mathrm{e}}=z_{10} \exp \left(-k_{\infty} U_{10}\right) .
$$

The considered spray-laden flow configuration is schematically shown in Fig. 1.

\subsection{Estimates for Values of the Parameters Characterizing the Problem}

Three non-dimensional parameters $s_{0}, U_{\mathrm{W}}$ and $\omega$ occur in the governing equations and boundary conditions (35)-(40). We will also use an alternative equivalent set of parameters $s_{0}, u_{*}$ and $\alpha_{\mathrm{s}}$. In order to proceed with our analysis, we need to estimate the range and limiting values of these parameters. As mentioned above, the value of $s_{0}$ is defined by the spray-generation function (SGF) via relationship (11). A comprehensive review of the SGFs for low wind speeds is given in Andreas (1998), where it is noted that a great uncertainty regarding the SGF magnitude exists in literature. Its estimations suggested by different authors vary by six orders of magnitude for a given droplet radius even for low wind speeds, and currently no reliable experimental data are available for high wind speeds. Many researchers in the field (Wu 1993; Andreas 1998; Monahan 1984), however, suggest that the spume ocean-spray production increases exponentially with the flow speed at the reference level $u_{10}$, i.e.

$$
s_{0}=A \exp \left(\delta\left(u_{10}-u_{\mathrm{r}}\right)\right),
$$

where $A=2 \times 10^{-7}$ and the reference speed $u_{\mathrm{r}}=22 \mathrm{~m} \mathrm{~s}^{-1}$. In order to be consistent with the SGFs reported in the previous studies, we take the value of $u_{10}$ in (44) without accounting for the flow acceleration due to the spray presence. The SGF values for the actual flow speed would be somewhat lower due to the spray lubrication effect. For the present, we consider the lower- and upper-bound SGFs corresponding to the parameter $\delta$ (which should not be confused with the Dirac delta function introduced in Sect. 2) estimated (for $u_{10}$ in $\mathrm{m} \mathrm{s}^{-1}$ ) as 0.2 (Andreas 1998) and 0.875 (Monahan 1984; Wu 1993), respectively (both estimations 
(a)

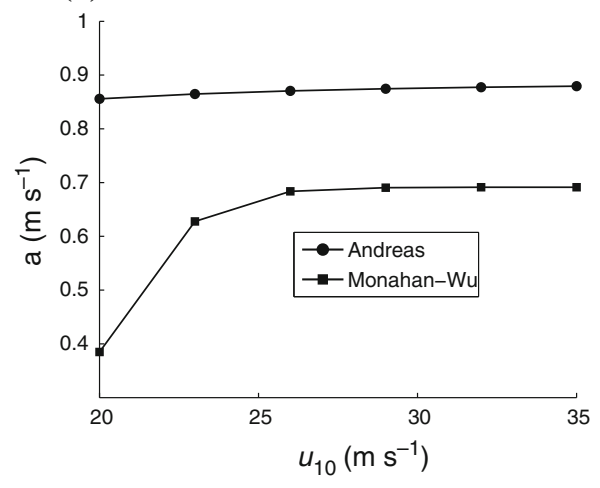

(b)

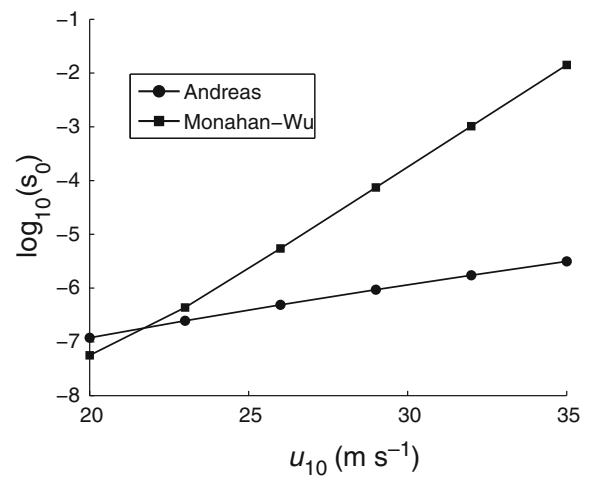

Fig. 2 a Average terminal velocity of a droplet and $\mathbf{b}$ logarithm of the spray volume mixing ratio $s_{0}$ versus the reference flow speed $u_{10}$. The terminal velocities and spray volume mixing ratio are calculated for spray generation suggested by Andreas (1998) (circles) and Monahan (1986) with the spume-component modification by Wu (1993) (squares)

account for the spume and whitecap contributions). The values of the logarithm of $s_{0}$ and $a$ below $z \mathrm{~W}$ given by (11) and (15) are shown in Fig. 2 as functions of the reference flow speed $u_{10}$ for both SGFs. The average terminal velocity of spray droplets reaches the limiting value at wind speeds $u_{10}>25 \mathrm{~m} \mathrm{~s}^{-1}$ when the whitecap spray production becomes negligible compared to the spume spray production. The limiting values computed for different spray production rates remain of the order of $1 \mathrm{~m} \mathrm{~s}^{-1}\left(a \approx 0.9 \mathrm{~m} \mathrm{~s}^{-1}\right.$ for $\delta=0.2$ and $a \approx 0.7 \mathrm{~m} \mathrm{~s}^{-1}$ for $\delta=0.875)$. In contrast, the volume mixing ratio of the ocean spray depends strongly on the spray-generation rate and increases with the wind speed much faster for $\delta=0.875$ than for $\delta=0.2$. For example, at $u_{10}=35 \mathrm{~m} \mathrm{~s}^{-1}$, the volume mixing ratio of the spray is $s_{0} \approx 10^{-6}$ for $\delta=0.2$ and $s_{0} \approx 10^{-4}$ for $\delta=0.875$.

The parameter $\alpha_{\mathrm{s}}$ is the reciprocal of the turbulent Schmidt number $\alpha_{\mathrm{s}}=1 / S c_{\mathrm{t}}$, where the values of $S c_{\mathrm{t}}$ for atmospheric flows are not well known. For example, the average value of $S c_{\mathrm{t}}=0.6$ with the standard deviation of 0.31 was found by measuring the tracer mass emission in an atmospheric boundary layer near the ground (Flesch 2002). A similar range $S c_{\mathrm{t}}=0.3-0.9$ was reported by Jha and Bombardelli (2009) for sediment-laden open-channel flows. Other experimental studies report the estimated $S c_{\mathrm{t}}$ values varying widely from 0.17 to 1.35 (Lilly 1973; Koeltzsch 2000; Tominaga and Stathopoulos 2007). In our calculations, we consider the Schmidt number range of $0.3-1.0$ that corresponds to $\alpha_{\mathrm{s}} \approx 1-3$. Finally, we assume the average wave height of $10 \mathrm{~m}$ with the corresponding average wave amplitude $z_{\mathrm{W}}=5 \mathrm{~m}$.

\section{Numerical and Analytical Calculations Using the Model}

\subsection{Numerical Calculations}

In what follows, we discuss the results of numerical simulations of the spray-laden flow described by the two suggested models: the TKE-based model (35)-(37) and the MOSbased model (22)-(23) subject to the same boundary conditions (39) and (40). In order to investigate the sensitivity of the TKE model to Schmidt number values, we performed 

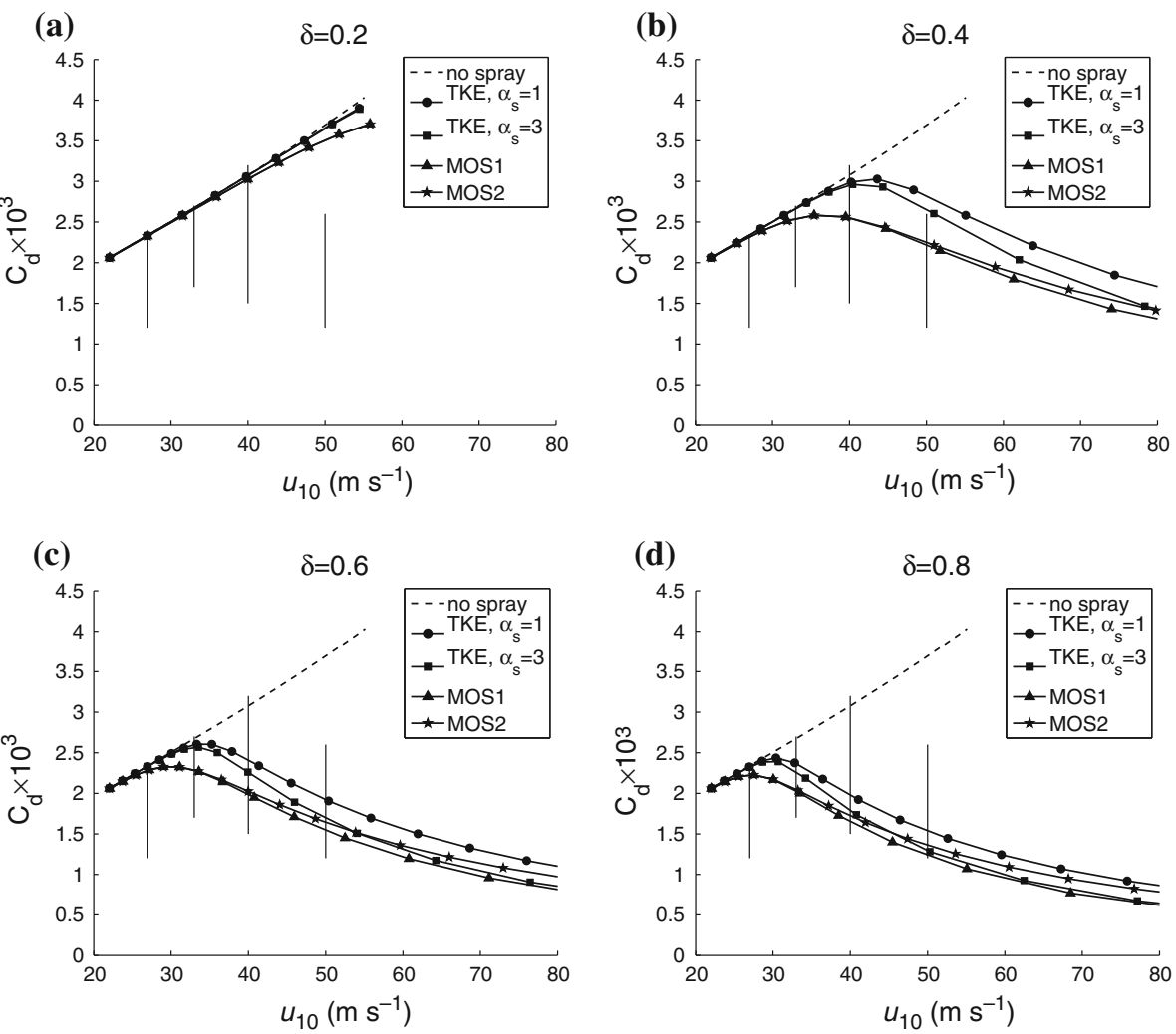

Fig. 3 Drag coefficient $C_{\mathrm{d}}$ as a function of the reference wind speed $u_{10}$ for $\mathbf{a} \delta=0.2, \mathbf{b} \delta=0.4$, $\mathbf{c} \delta=0.6$ and $\mathbf{d} \delta=0.8$. The circles and squares correspond to the TKE model with $\alpha_{\mathrm{S}}=1$ and $\alpha_{\mathrm{S}}=3$, respectively. The triangles and stars correspond to the MOS1 (SHEMA stability functions) and MOS2 (log-linear stability functions) models, respectively

calculations for $\alpha_{\mathrm{s}}=1$ and $\alpha_{\mathrm{s}}=3$. The results for the MOS model that uses SHEBA functions (28)-(29) and the log-linear distribution (26) are referred to as 'MOS1' and 'MOS2', respectively. The values of $\delta=0.2$ and $\delta=0.875$ correspond to the lower and upper bounds of the investigated spray production rate. The numerical results are compared with the measured data ranges given in Powell et al. (2003) and shown by the vertical bars in Figs. 3, 4 and 5. We also present the numerical data for the turbulent boundary-layer flow without spray (the dashed lines in Figs. 3, 4 and 5).

The values of the $10-\mathrm{m}$ drag coefficient $C_{\mathrm{d}}$ versus the 10 -m flow speed are plotted in Fig. 3. The TKE and MOS-based models predict qualitatively similar dependences of the drag coefficient on the flow speed over the considered range of parameters. The momentum transport reduction is insignificant until the flow speed reaches the critical value $u_{\mathrm{c}}$ (formally defined in the next sub-section). The critical value $u_{\mathrm{c}}$ decreases from $40-60 \mathrm{~m} \mathrm{~s}^{-1}$ for $\delta=0.2$ to $25-33 \mathrm{~m} \mathrm{~s}^{-1}$ for $\delta=0.8$. The MOS1 and MOS2 models produce even more closely similar dependences of the drag coefficient on the flow speed despite the significant difference between the log-linear and SHEBA stability functions. The values of $\xi$ and $R i$ at the wave crest level for $u_{10}=50 \mathrm{~m} \mathrm{~s}^{-1}$ correspond to the maxima of these variables over the observed flow-speed interval. They are presented in Table 1. These numerical results 

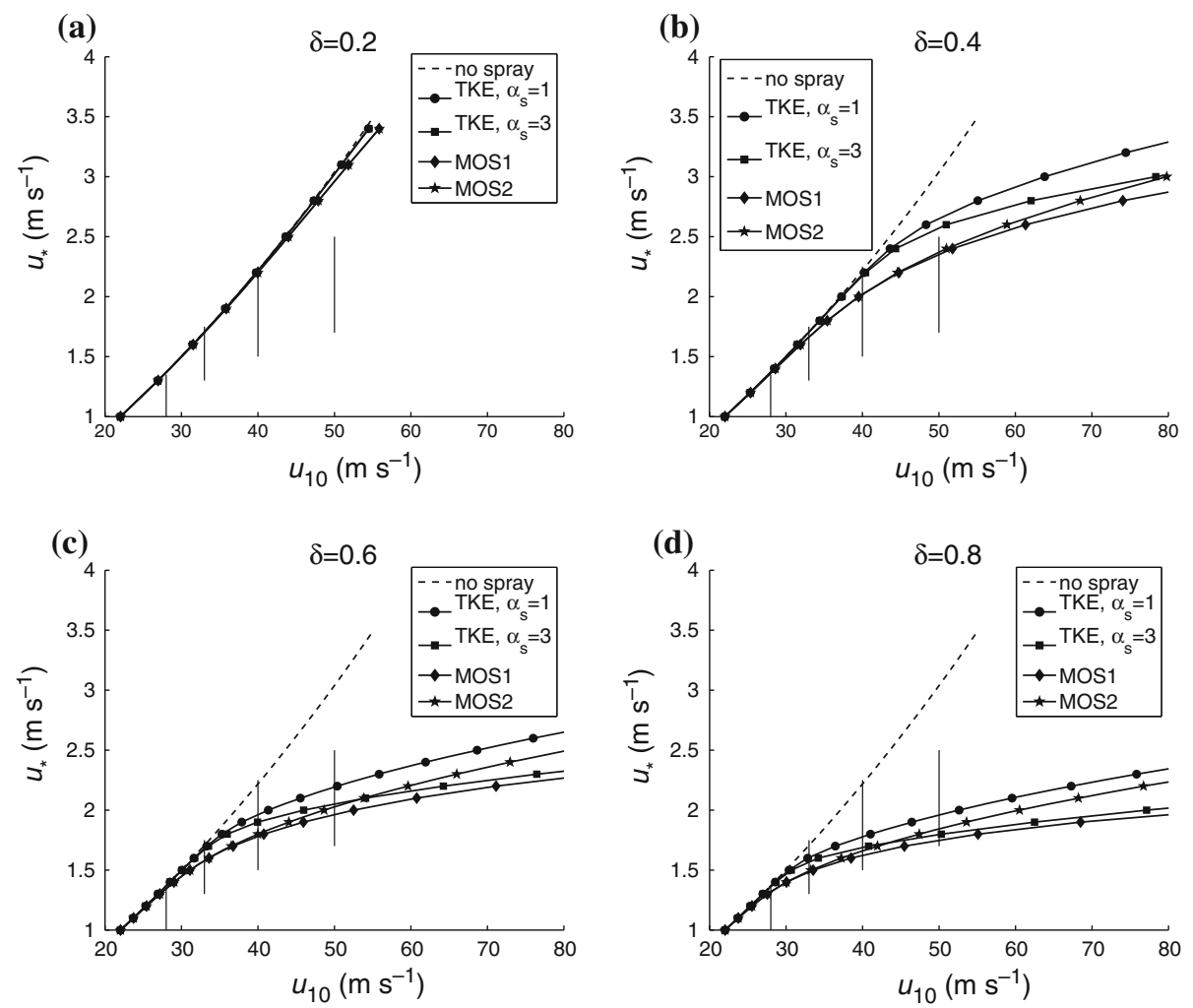

Fig. 4 Friction velocity $u_{*}$ as a function of the reference wind speed $u_{10}$ for the same parameters as in Fig. 3

indicate that almost all considered cases fall into either weakly stable or transitional regimes with $R i \lesssim R i_{\mathrm{c}} \approx 0.2$, safely in the validity range of MOS theory. The TKE model predicts a stronger lubrication for larger values of $\alpha_{\mathrm{S}}$ (smaller turbulent Schmidt number) but the same value of the critical flow speed. In comparison with the TKE model, the MOS models predict a stronger drag reduction at larger flow speeds and lower values of the critical speed at which the lubrication effects become noticeable, especially for smaller values of $\delta=0.2-0.4$.

Figure 4 shows the dependence of the friction velocity $u_{*}$ on the $10-\mathrm{m}$ flow speed, where good quantitative agreement amongst all the models is observed. The numerical results are relatively insensitive to the value of the turbulent Schmidt number and the choice of the momentum and spray-flux stability functions. The dependence of the effective roughness length $z_{0 \mathrm{e}}$ defined by (43) on the wind speed is presented in Fig. 5. For the same wind speed, the TKE models predict a larger effective turbulent roughness than do the MOS models. Good agreement between numerical simulations and measurements is observed for larger values of $\delta$ in the range of $0.4-0.8$ for the MOS models and $0.5-0.8$ for the TKE model. However, the numerical results obtained for $\delta=0.2$ differ from the experimental observations. The best match with the experimental data is obtained for $\delta=0.5-0.6$, which is in the middle of the $\delta$ value range found in the literature. For these values of $\delta$, the product $\sigma s$ becomes larger than unity for $u_{10}>40 \mathrm{~m} \mathrm{~s}^{-1}$. As we mentioned above, for such large wind speeds, the spray inertia might become important so that it is unclear whether the inertialess TKE model remains valid in these regimes. On the other hand, the MOS1 model is still formally 

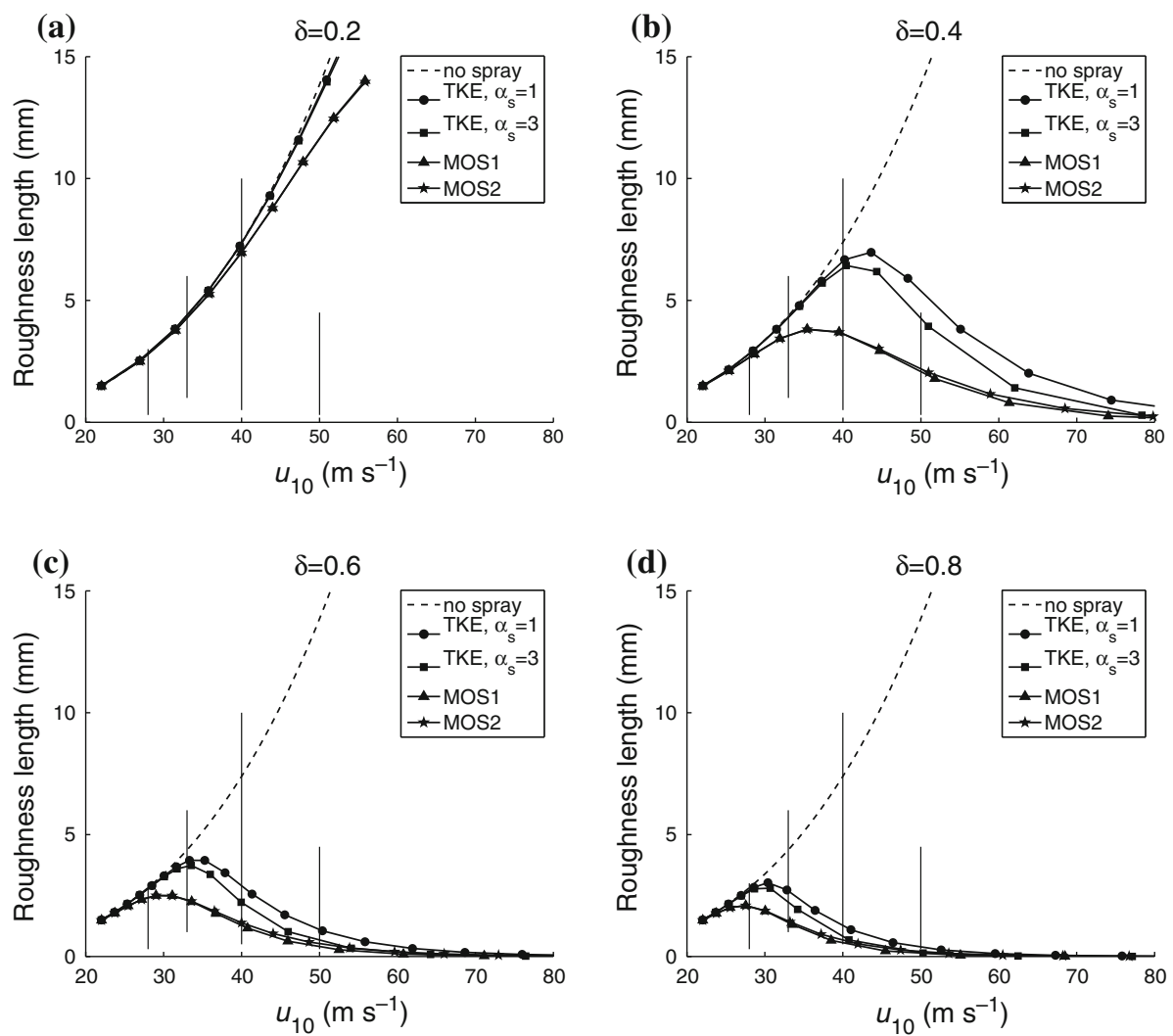

Fig. 5 Effective roughness length as a function of the reference wind speed $u_{10}$ for the same parameters as in Fig. 3

valid so long as $s<10^{-3}$ and the fact that both models produce similar results serves as an indicator that the current TKE model captures the lubrication effect quite accurately even for larger values of spray concentration.

\subsection{Analytical Estimates}

In this section, we describe the asymptotic solution for a spray-laden wind. It is valid for small values of Kolmogorov number $K o$, implying a small spray volume mixing ratio. Therefore, the asymptotic solution describes the initial stage of flow acceleration when the spray concentration is low. This solution allows one to evaluate the critical flow speed $u_{\mathrm{c}}$.

Since $K o \geq 0$ it follows from (37) that

$$
\sqrt{E}=(1-K o)^{1 / 4} \leq 1
$$

and from (45) and (38), we obtain

$$
K o \leq k_{\infty} \omega L s \leq \frac{k_{\infty}^{2} z}{L_{\mathrm{r}}} \omega s_{0}=\frac{k_{\infty} z s_{0} a \sigma g}{u_{*}^{3}} .
$$


Taking $z \approx 10 \mathrm{~m}, a \approx 1 \mathrm{~ms}^{-1}, \sigma \approx 10^{3}, g \approx 10 \mathrm{~ms}^{-2}, u_{*} \approx 1 \mathrm{~ms}^{-1}$, we obtain an estimate:

$$
K o \lesssim 0.1,
$$

which is valid for low spray concentrations $s_{0} \lesssim 10^{-6}$. Therefore, $K o$ is a small parameter for this range of $s_{0}$ values. After expanding the expression for the reciprocal of the nondimensional turbulent diffusion coefficient $K$ into a Taylor series in $K o$, and retaining only first-order terms, we obtain

$$
\frac{1}{K}=\frac{1}{\sqrt{E} L}=\frac{1}{(1-K o)^{1 / 4} L}=\frac{(1+K o / 4+\cdots)}{L} \approx \frac{\left(1+\frac{k_{\infty} \omega L s}{4}\right)}{L} .
$$

Upon substituting (48) into (36) and disregarding the higher-order terms containing $K o$, we obtain the following equation for $s$ :

$$
\frac{\mathrm{d} s}{\mathrm{~d} Z}+\frac{\omega}{Z} s=0
$$

subjected to the boundary condition

$$
s\left(Z_{\mathrm{W}}\right)=s_{0} .
$$

The solution of (49) and (50) can be written as

$$
s(Z)=s_{0}\left(\frac{Z}{Z_{\mathrm{W}}}\right)^{-\omega} .
$$

After substituting (48) into (35) and integrating the obtained equation with respect to the vertical coordinate $Z$, we obtain the expression for the non-dimensional flow speed as follows:

$$
U=\frac{1}{k_{\infty}} \ln \left(\frac{Z}{Z_{0}}\right)+\frac{1}{4} k_{\infty} \omega Z_{\mathrm{W} s_{0}} I(Z),
$$

where

$$
I(Z)=\left\{\begin{array}{ll}
\frac{\left(\frac{Z}{Z_{\mathrm{W}}}\right)^{1-\omega}-1}{1-\omega}, & \omega \neq 1 \\
\ln \left(\frac{Z}{Z_{\mathrm{W}}}\right), & \omega=1
\end{array} .\right.
$$

The first term on the right-hand side of $(52), U^{0}=\left(1 / k_{\infty}\right) \ln \left(Z / Z_{0}\right)$, is the non-dimensional horizontal speed profile for a flow without spray, while the second term determines the velocity magnitude increase due to the presence of the spray.

After substituting the expression for the spray concentration (44) into (52) we obtain

$$
u=u^{0}+I(z) C \exp \left(\delta\left(u_{10}^{0}-u_{\mathrm{r}}\right)\right),
$$

where $u$ and $u^{0}$ are the dimensional flow velocities corresponding to $U=u / u_{*}, U^{0}=u^{0} / u_{*}$ and $C=k_{\infty} \omega Z_{\mathrm{W}} A u_{*} / 4$. At the reference point $z_{10} \equiv 10 \mathrm{~m}$, Eq. (54) becomes

$$
u_{10}=u_{10}^{0}+D \exp \left(\delta\left(u_{10}^{0}-u_{\mathrm{r}}\right)\right)
$$

where $D=I\left(z_{10}\right) C$. The obtained relationship (55) allows us to estimate the critical value of the flow speed at which the airflow acceleration 'kicks in'. 
We define the critical flow speed $u_{\mathrm{c}}$ as that for which the difference between the sprayladen flow speed and that without the spray at the reference level $z_{10}$ is equal to $1 \mathrm{~m} \mathrm{~s}^{-1}$ i.e. $u_{\mathrm{c}}=u_{\mathrm{c}}^{0}-1$. This implies that, according to (55),

$$
D \exp \left(\delta\left(u_{\mathrm{c}}-u_{\mathrm{r}}\right)\right)=1 .
$$

Finally, we obtain the estimate for the critical speed $u_{\mathrm{c}}$ from (56):

$$
u_{\mathrm{c}}^{0} \approx u_{\mathrm{c}}=u_{\mathrm{r}}-\frac{\ln D}{\delta}=u_{\mathrm{r}}-\frac{1}{\delta} \ln \left(\frac{a z_{\mathrm{W}} A \sigma g}{4 u_{*}^{3}} I\left(z_{10}\right)\right) .
$$

In particular, the critical flow speed $u_{\mathrm{c}}=54,38,33$, and $30 \mathrm{~m} \mathrm{~s}^{-1}$ for $\delta=0.2,0.4,0.6$ and 0.8 , respectively, and $u_{*}=1 \mathrm{~m} \mathrm{~s}^{-1}, z_{\mathrm{W}}=5 \mathrm{~m}, \alpha_{\mathrm{s}}=1$. The value of $u_{*}$ varies somewhat with $\delta$, but, since it only appears logarithmically, its effect on the estimate is insignificant. The analytical values of $u_{\mathrm{c}}$ agree very well with the numerical results (refer to Fig. 3).

\subsection{Remarks on the BCP Mathematical Model}

While the current study was strongly influenced and inspired by the study reported in BCP, we believe that BCP contains a number of physical and numerical shortcomings that are outlined below. The BCP model considers ocean spray generated by the wind tearing droplets off wave crests within the spume layer located between $z_{0}$ and $z_{\mathrm{w}}=z_{0}+h$. The following main result is reported by the authors in their Discussion section: "An illustrative numerical example is as follows: with standard values of the water and air densities, $u_{*}=1 \mathrm{~m} \mathrm{~s}^{-1}, z_{\mathrm{W}}=10 \mathrm{~m}, z_{0}=9.9 \mathrm{~m}, h=0.1 \mathrm{~m}$, the wind speed with no droplets at $z=20 \mathrm{~m}$ is $\approx 4 \mathrm{~m} \mathrm{~s}^{-1}$, while in the presence of large droplets at $s_{0}=10^{-4}, \omega=75$, the wind speed is $30 \mathrm{~m} \mathrm{~s}^{-1}$ ". However, the reported value $\omega=75\left(\omega\right.$ is defined as $\left.\omega=a /\left(\alpha_{\mathrm{s}} u_{*}\right)\right)$ corresponds to the terminal velocity $a=\alpha_{\mathrm{s}} u_{*} \omega=150 \mathrm{~m} \mathrm{~s}^{-1}$, which is unrealistically large. For comparison, in the classical study of Gunn and Kinzer (1949), the terminal velocity of a droplet with the diameter of $5.8 \mathrm{~mm}$ is found to be only $9.17 \mathrm{~m} \mathrm{~s}^{-1}$. The value of the surface roughness $z_{0}=9.9 \mathrm{~m}$ also appears to be very large. Despite these physical inconsistencies, we repeated the BCP calculations using their model and parameter values. However, we found that the flow speed increased only by $3 \%$ from 2.5 to $2.57 \mathrm{~m} \mathrm{~s}^{-1}$ in contrast to the increase of almost an order of magnitude reported in BCP. It is likely that numerical errors in the BCP calculations are responsible for this large discrepancy.

\section{Conclusions}

We proposed two (MOS and TKE-based) models for the effect of ocean spray on the momentum exchange at high wind speeds. Primarily the spume spray that results from wave crests torn by the wind affects the vertical momentum exchange. The contribution of jet and film droplets in the momentum exchange rate is negligible since its total volume production at wind speeds $>20 \mathrm{~m} \mathrm{~s}^{-1}$ is much lower than that of spume droplets.

It is found that the airflow in the lower part of the boundary layer can noticeably accelerate because of the turbulent transport suppression by the ocean spray if the spray concentration increases rapidly enough with the wind speed. The lubrication effect is insignificant until the flow speed reaches a certain critical value. Further wind speed increases trigger flow acceleration relative to the airflow not affected by spume. It is estimated that, depending on the exponential spume-generation growth rate, the critical wind speed ranges from $25-33 \mathrm{~m} \mathrm{~s}^{-1}$ 
(for a large growth rate reported by Wu 1993) to more than $45 \mathrm{~m} \mathrm{~s}^{-1}$ (for the slowest growth rate reported by Andreas 1998).

The applicability of the considered MOS theory (unlike the developed TKE model) for the atmospheric spray-laden boundary layer is not warranted since the original MOS model was developed using stability functions experimentally determined for a thermally stratified boundary layer. However, as we pointed out, there is no full analogy between thermally and sediment-stratified boundary layers. Yet, the numerical results indicate that both the MOS and TKE models produce similar distributions for a complete range of flow parameters. Therefore, we conclude that utilization of the MOS theory for a spray-laden boundary-layer flow analysis leads to results that are at least qualitatively correct.

Numerical calculations have been verified against the available experimental data (Powell et al. 2003). It was shown that the dependencies of the drag coefficient, friction velocity and efficient roughness length on the flow speed agree well with the corresponding experimental data. Therefore, the spray stratification of the boundary layer can explain the significant reduction of momentum transport between the atmosphere and the ocean under high wind conditions observed experimentally.

We also re-examined the findings reported in Barenblatt et al. (2005) in which the authors argued that the presence of water spray can significantly accelerate the flow. While our investigation was inspired by this idea and fully supports it, we believe that the numerical results reported there might be affected by computational errors and an unrealistic choice of model parameters.

Acknowledgments The study of YR and YLL was supported by a grant from the National Oceanic and Atmospheric Administration, Educational Partnership Program under the cooperative agreement NA06OAR4810187.

Open Access This article is distributed under the terms of the Creative Commons Attribution Noncommercial License which permits any noncommercial use, distribution, and reproduction in any medium, provided the original author(s) and source are credited.

\section{References}

Andreas EL (1998) A new sea spray generation function for wind speeds up to $32 \mathrm{~m} / \mathrm{s}$. J Phys Oceanogr 28:2175-2184

Andreas EL, DeCosmo J (1999) See spray production and influence on air-sea heat and moisture fluxes over the open ocean (Chapter 13). In: Geernaert GL (ed) Air-sea exchange: physics, chemistry and dynamics. Kluwer, Dordrecht, pp 327-362

Andreas EL, Emanuel KA (2001) Effects of sea spray on tropical cyclone intensity. J Atmos Sci 58:3741-3751

Andreas EL, Edson JB, Monahan EC, Rouault MP, Smith SD (1995) The spray contribution to the evaporation from the sea: a review of recent progress. Boundary-Layer Meteorol 72:3-52

Anguelova M, Barber RP Jr, Wu J (1999) Spume drops produced by the wind tearing of wave crests. J Phys Oceanogr 29:1156-1165

Babanin AV, Makin VK (2008) Effects of wind trend and gustiness on the sea drag: Lake George study. J Geophys Res 113:C02015. doi:10.1029/2007JC004233

Bao JW, Wilczak JM, Choi JK, Kantha LH (2000) Numerical simulations of air-sea interaction under high wind conditions using a coupled model: a study of hurricane development. Mon Weather Rev 128: 2190-2210

Barenblatt GI (1953) On the motion of suspended droplets in a turbulent flow. Prikl Mat Mekh 17(3):261-274 (in Russian)

Barenblatt GI (1996) Scaling, self-similarity, and intermediate asymptotics: dimensional analysis and intermediate asymptotics. Cambridge University Press, Cambridge, 412 pp

Barenblatt GI, Golitsyn GS (1974) Local structure of mature dust storms. J Atmos Sci 31(7):1917-1933 
Barenblatt GI, Chorin AJ, Prostokishin VM (2005) A note concerning the Lighthill "sandwich model" of tropical cyclones. Proc Natl Acad Sci USA 102(32):1115-1148

Beljaars ACM, Holtslag AAM (1991) Flux parameterization over land surfaces for atmospheric models. J Appl Meteorol 30(3):327-341

Blanchard DC (1963) The electrification of the atmosphere by particles from bubbles in the sea. In: Sears M (ed) Progress in oceanography, vol 1. MacMillan, New York, pp 71-202

Borisenkov EP (1974) Some mechanisms of atmosphere-ocean interaction under stormy weather conditions. Probl Arct Antarct 43-44, 73-83 (in Russian)

Bortkovskii RS (1973) On the mechanism of interaction between the ocean and the atmosphere during a storm. Fluid Mech Sov Res 2:87-94

Bortkovskii RS (1987) Air-sea exchange of heat and moisture during storms. D. Reidel Publishing, Boston, $194 \mathrm{pp}$

Businger JA, Yaglom AM (1971) Introduction to Obukhov's paper on 'turbulence in an atmosphere with a non-uniform temperature'. Boundary-Layer Meteorol 2:3-6

Businger JA, Wyngaard JS, Izumi Y, Bradley EF (1971) Flux-profile relationships in the atmospheric surface layer. J Atmos Sci 28:181-189

Charnock H (1955) Wind stress on a water surface. Q J R Meteorol Soc 81:639-640

De Leeuw G (1986) Vertical profiles of giant particles close above the sea surface. Tellus 38B:51-61

De Leeuw G (1993) Aerosols near the air-sea interface. Trends Geophys Res 2:55-70

Donelan MA, Haus BK, Reul N, Plant WJ, Stiassnie M, Graber HC, Brown OB, Saltzman ES (2004) On the limiting aerodynamic roughness of the ocean in very strong winds. Geophys Res Lett 31:L18306. doi:10. 1029/2004GL019460

Dyer AJ (1974) A review of flux-profile relationships. Boundary-Layer Meteorol 7:363-372

Fairall CW, Kepert JD, Holland GJ (1994) The effect of sea spray on surface energy transports over the ocean. Glob Atmos Ocean Syst 2:121-142

Fairall CW, Banner ML, Peirson WL, Asher W, Morison RP (2009) Investigation of the physical scaling of sea spray spume droplet production. J Geophys Res 114:C10001. doi:10.1029/2008JC004918

Flesch TK (2002) Turbulent Schmidt number from a tracer experiment. Agric For Meteorol 111:299-307

Gall JS, Frank WM, Kwon Y (2008) Effects of sea spray on tropical cyclones simulated under idealized conditions. Mon Weather Rev 136:1686-1705

Gathman SG (1982) A time-dependent oceanic aerosol profile model. NRL Rep. 8536, Naval Research Laboratory, Washington, DC (NTIS AD-A111148) 35 pp

Grachev AA, Fairall CW, Persson POG, Andreas EL, Guest PS (2005) Stable boundary-layer scaling regimes: the SHEBA data. Boundary-Layer Meteorol 116(2):201-235

Grachev AA, Andreas EL, Fairall CW, Guest PS, Persson POG (2007) SHEBA flux-profile relationships in the stable atmospheric boundary layer. Boundary-Layer Meteorol 124(3):315-333

Grachev AA, Andreas EL, Fairall CW, Guest PS, Persson POG (2008) Turbulent measurements in the stable atmospheric boundary layer during SHEBA: ten years after. Acta Geophys 56(1):142-166

Gunn R, Kinzer GD (1949) The terminal velocity of fall for water droplets in stagnant air. J Meteorol 6: 243-248

Holtslag AAM, de Bruin HAR (1988) Applied modelling of the nighttime surface energy balance over land. J Appl Meteorol 27:689-704

Howell JF, Sun J (1999) Surface-layer fluxes in stable conditions. Boundary-Layer Meteorol 90:495-520

Iida N, Toba Y, Chaen M (1992) A new expression for the production rate of sea water droplets on the sea surface. J Oceanogr 48:439-460

Jha SK, Bombardelli FA (2009) Two-phase modelling of turbulence in dilute sediment-laden. open-channel flows. Environ Fluid Mech 9:237-266

Kepert J, Fairall C, Bao J-W (1999) Modelling the interaction between the atmospheric boundary layer and evaporating sea spray droplets (Chapter 14). In: Geernaert GL (ed) Air-sea exchange: physics, chemistry and dynamics. Kluwer, Dordrecht, pp 63-409

King JC (1990) Some measurements of turbulence over an Antarctic shelf. Q J Roy Meteorol Soc 116:379-400

Koeltzsch K (2000) The height dependence of the turbulent Schmidt number within the boundary layer. Atmos Environ 34:1147-1151

Kolmogorov AN (1954) New variant of the gravitational theory of motion of suspended sediment. Vestnik MGU 3:41-45 (in Russian)

Kudryavtsev VN (2006) On the effect of sea drops on the atmospheric boundary layer. J Geophys Res 111:C07020. doi:10.1029/2005JC002970

Lai RJ, Shemdin OH (1974) Laboratory study of the generation of spray over water. J Geophys Res 79(21):3055-3063 
Lettau HH (1957) Summary of non-dimensional characteristics of boundary layer theory. In: Lettau HH, Davidson B (eds) Exploring the atmosphere's first mile, vol 1. Pergamon Press, New York, pp 337-372

Lighthill J (1999) Ocean spray and the thermodynamics of tropical cyclones. J Eng Math 35:11-49

Lilly GP (1973) Effect of particle size on particle eddy diffusivity. Ind Eng Chem Fundam 12(3):268-275

Ling SC, Kao TW (1976) Parameterization of the moisture and heat transfer process over the ocean under whitecap sea states. J Phys Oceanogr 6(3):306-315

Ling SC, Kao TW, Saad AI (1980) Microdroplets and transport of moisture from ocean. Proc ASCE Eng Mech Div 106:1327-1339

Mahrt L (1998) Stratified atmospheric boundary layers. Boundary-Layer Meteorol 90(3):375-396

Makin VK (2004) A note on the drag of the sea surface at hurricane winds. Boundary-Layer Meteorol 115(1):169-176

Monahan EC (1986) The ocean as a source for atmospheric particles. In: Buat-Menard P (ed) The role of air-sea exchange in geochemical cycling. D Reidel, Norwell, pp 129-163

Monin AS, Yaglom AM (2007) Statistical fluid mechanics, volume I: mechanics of turbulence. Dover Publications, Mineola New York, USA, 784 pp

Nieuwstadt FTM (1984) The turbulent structure of the stable, nocturnal boundary layer. J Atmos Sci 41: 2202-2216

Powell MD, Vickery PJ, Reinhold TA (2003) Reduced drag coefficient for high wind speeds in tropical cyclones. Nature 422:279-283

Smith MH, Park PH, Consterdine IE (1993) Marine aerosol concentrations and estimated fluxes over the sea. Q J R Meteorol Soc 119:809-824

Soulsby RL, Wainwright BLSA (1987) A criterion for the effect of suspended sediment on near-bottom velocity profiles. J Hydraul Res 25(3):341-356

Tennekes H (1973) The logarithmic wind profile. J Atmos Sci 30:234-238

Tominaga Y, Stathopoulos T (2007) Turbulent Schmidt numbers for CFD analysis with various types of flow field. Atmos Environ 41:8091-8099

Vakhguelt A (2007) Mathematical model of interaction between finite amplitude wave and the liquid particles. In: Proceedings of the 18th Australasian coastal and ocean engineering conference and the 11th Australasian port and harbour conference, 18-20 July 2007, Melbourne, VIC, paper 197

Wamser C, Lykossov VN (1995) On the friction velocity during blowing snow. Beitr Phys Atmos 68(1):85-94

Wang Y, Kepert GD, Holland GJ (2001) The effect of sea spray evaporation on tropical cyclone boundary layer structure and intensity. Mon Weather Rev 129:2481-2500

Woolf DK, Bowyer PA, Monahan EC (1987) Discriminating between the film drops and jet drops produced by a simulated whitecap. J Geophys Res 92:5142-5150

Wu J (1979) Spray in the atmospheric surface layer: review and analysis of laboratory and oceanic results. J Geophys Res 84(NC4):1693-1704

Wu J (1980) Wind stress coefficients over sea surface near neutral conditions_-a revisit. J Phys Oceanogr 10:727-740

Wu J (1992) Individual characteristics of whitecaps and volumetric description of bubbles. IEEE J Ocean Eng 17(1):150-158

Wu J (1993) Production of spume drops by the wind tearing of wave crests: the search for quantification. J Geophys Res 98:18221-18227

Yaglom AM (1977) Comments on wind and temperature flux-profile relationships. Boundary-Layer Meteorol 11:89-102 